\title{
Detection of Reactive Oxygen Species Using MitoSOX and CellROX Green in Zebrafish
}

Alberto Rissone ${ }^{1,2, *}$ and Fabio Candotti ${ }^{2,3, *}$

\begin{abstract}
${ }^{1}$ Translational and Functional Genomics Branch, National Human Research Institute (NHGRI), National Institutes of Health (NIH), Bethesda, MD, USA; ${ }^{2}$ Disorders of Immunity Section, Genetics and Molecular Biology Branch, NHGRI, NIH, Bethesda, MD, USA; ${ }^{3}$ Division of Immunology and Allergy, University Hospital of Lausanne, Lausanne, Switzerland

*For correspondence: alberto.rissone@nih.gov; Fabio.Candotti@chuv.ch
\end{abstract}

[Abstract] The production of free radicals is the result of normal cellular metabolism. Free radicals are involved in innumerable different cellular and biological functions such as signaling, proliferation, cell death, aging, inflammation, etc. Under physiological conditions, the levels of reactive oxygen species (ROS) are strictly regulated by the cells. However, during stressful conditions such as oxidative stress, ROS levels increase causing damages to different molecules like DNA, lipids and proteins. Increased levels of ROS have been associated with a growing list of different diseases. In this protocol, we used MitoSOX and CellROX Green oxidative stress probes to label the intracellular ROS and detect the fluorescence using cell sorting and confocal analyses.

\section{Materials and Reagents}

1. $10 \mathrm{~cm}$ sterile Petri dishes (Thermo Fisher Scientific, Fisher Scientific, catalog number: FB0875711Z)

2. $1.5 \mathrm{ml}$ tubes (Eppendorf, catalog number: 022363204)

3. $35 \mathrm{~mm}$ TC-Treated culture dish (Corning, catalog number: 430165)

4. Falcon round-bottom polystyrene $5 \mathrm{ml}$ tubes (Corning, Falcon ${ }^{\circledR}$, catalog number: 352003 )

5. Sterile $40 \mu \mathrm{m}$ cell strainer (Corning, catalog number: 431750)

6. $35 \mathrm{~mm}$ glass bottom dish (Cellvis, catalog number: D35-20-1-N)

7. $3 \mathrm{ml}$ transfer pipette (Sigma-Aldrich, catalog number: Z135003)

8. Zebrafish adults (wild-type or mutant strains)

9. Sodium chloride ( $\mathrm{NaCl}$ ) (Sigma-Aldrich, catalog number: S7653)

10. Potassium chloride (KCl) (Sigma-Aldrich, catalog number: P9333)

11. Calcium chloride dihydrate $\left(\mathrm{CaCl}_{2} \cdot 2 \mathrm{H}_{2} \mathrm{O}\right)$ (Sigma-Aldrich, catalog number: $\left.\mathrm{C} 5080\right)$

12. Magnesium sulfate heptahydrate $\left(\mathrm{MgSO}_{4} \cdot 7 \mathrm{H}_{2} \mathrm{O}\right)$ (Sigma-Aldrich, catalog number: 63138)

13. $\mathrm{dd}_{2} \mathrm{O}$ (Sigma-Aldrich, catalog number: W4502)

14. DMEM/F-12 medium (Thermo Fisher Scientific, Gibco ${ }^{\mathrm{TM}}$, catalog number: 11330-032)

15. Fetal bovine serum (FBS) (Thermo Fisher Scientific, Gibco ${ }^{T M}$, catalog number: 16000-036)

16. Tricaine (Sigma-Aldrich, catalog number: A5040) 
17. 10x trypsin-EDTA (0.5\%), no phenol red (Thermo Fisher Scientific, Gibco ${ }^{\mathrm{TM}}$, catalog number: 15400-054)

18. Tris-EDTA buffer solution (pH 9.0) (Sigma-Aldrich, catalog number: SRE0063)

19. Collagenase $P$ (Sigma-Aldrich, catalog number: COLLP-RO)

20. Phosphate buffered saline (PBS), pH 7.4 (Thermo Fisher Scientific, Gibco ${ }^{\mathrm{TM}}$, catalog number: 10010-023)

21. Hank's balanced salt solution (HBSS), with calcium and magnesium, no phenol red (HBSS/Ca/Mg) (Thermo Fisher Scientific, Gibco ${ }^{\mathrm{TM}}$, catalog number: 14025-092)

22. MitoSOX ${ }^{\mathrm{TM}}$ red mitochondrial superoxide indicator (Thermo Fisher Scientific, Molecular Probes $^{\mathrm{TM}}$, catalog number: M36008)

23. Dimethyl sulfoxide (DMSO) (Sigma-Aldrich, catalog number: D2650)

24. 7-aminoactinomycin $D$ (7-AAD) (BD, BD Pharmingen ${ }^{\mathrm{TM}}$, catalog number: 559925)

25. Annexin V-APC (BD, BD Pharmingen ${ }^{\mathrm{TM}}$, catalog number: 550474)

26. Annexin $\vee$ binding buffer, 10x concentrate (BD, BD Pharmingen ${ }^{\mathrm{TM}}$, catalog number: 556454 )

27. CellROX ${ }^{\circledR}$ green reagents, for oxidative stress detection (Thermo Fisher Scientific, Molecular Probes $^{\mathrm{TM}}$, catalog number: $\left.\mathrm{C} 10444\right)$

28. CellROX ${ }^{\circledR}$ green flow cytometry assay kit (Thermo Fisher Scientific, Molecular Probes ${ }^{\top \mathrm{M}}$, catalog number: C10492)

29. Certified ${ }^{\mathrm{TM}}$ low-melting agarose (Bio-Rad Laboratories, catalog number: 1613111)

30. Instant Ocean aquarium sea salt mixture (Spectrum Brand, Instant Ocean, catalog number: SS15-10)

31. $24 x$ tricaine solution (see Recipes)

32. DMEM/F-12 medium (see Recipes)

33. 60x E3 embryo medium stock solution (see Recipes)

34. Homogenization medium (see Recipes)

35. $5 \mathrm{mM}$ MitoSOX stock solution (see Recipes)

\section{Equipment}

1. Dissecting forceps - microdissection (DR Instruments, catalog number: 18)

2. Ultrafine single deer hair with handle (Ted Pella, catalog number: 119)

3. Thermoblock (Sigma-Aldrich, catalog number: Z605271)

4. Manual pipette PR-200 (Mettler-Toledo, Rainin, catalog number: 17008652)

5. Standard tabletop centrifuges (Eppendorf, catalog number: 022620401)

6. Dissecting microscope (Leica, model: Leica S8 APO)

7. FACS-Fluorescence-activated cell sorter [e.g., FACSAria II equipped with FACSDiva software using the blue 488-nm and red 640-nm lasers (BD)] 
8. Confocal microscope [e.g., LSM 510 NLO Meta system mounted on an Axiovert 200M microscope (Carl Zeiss) with a Plan-Apochromat 10x/0.45 or a Plan-Apochromat 20x/0.75 objective lens]

\section{$\underline{\text { Software }}$}

1. FACSDiva software

\section{Procedure}

A. Single cell homogenization and ROS (MitoSOX and CellROX Green) staining

1. Grow zebrafish embryos at $28{ }^{\circ} \mathrm{C}$ in $\sim 25 \mathrm{ml}$ of fish water or $1 \mathrm{x} \mathrm{E} 3$ embryo medium until the desired stage.

2. Pre-heat the homogenization solution and the DMEM-10\% FBS at $28{ }^{\circ} \mathrm{C}$.

3. Collect the embryos and manually dechorionate them with a pair of forceps with sharp tips after -24 h post fertilization (hpf), if necessary. Use one forceps to hold the external membrane (chorion), then make a tear using the other forceps and gently remove the chorion.

4. Transfer dechorionated embryos to $1.5 \mathrm{ml}$ tubes using a plastic pipette.

Note: The quality of the homogenization will depend on the stage and the number of embryos used. Do not exceed the number of 50-60 embryos at 5 days post fertilization (dpf) embryos/tube.

5. Remove most of the water or $1 \times \mathrm{E} 3$ embryo medium without damaging or drying the embryos.

6. Euthanize embryos with $\sim 400 \mu \mathrm{l}$ of $24 \mathrm{x}$ tricaine stock solution and leave on ice for $5 \mathrm{~min}$.

7. Wash twice with $1 \mathrm{ml}$ of $1 \times$ PBS for 1-2 min.

8. Add $600 \mu \mathrm{l}$ of homogenization solution (collagenase $P+$ trypsin-EDTA in $1 \times$ PBS) and incubate at $28{ }^{\circ} \mathrm{C}$ in a thermoblock for 20-30 min depending on the developmental stage or until the complete homogenization of each sample.

9. During the incubation time, periodically ( every 1-2 $\mathrm{min}$ ) homogenate the samples pipetting up and down with a $200 \mu \mathrm{l}$ pipette. During the incubation time a progressive homogenization of the samples should be noted.

10. Add $700 \mu$ of DMEM-10\% FBS, mix by pipetting or vortexing and then centrifuge the cells for 2 $\min$ at $600 \times g$ at room temperature (RT).

11. Discard supernatant and resuspend the cells in $1 \mathrm{ml} 1 \times$ PBS.

12. Centrifuge the cells for 2 min at $600 \times g$.

13. Resuspend the cells in $1 \mathrm{ml}$ of DMEM-10\% FBS.

14. Keep the cells at $28^{\circ} \mathrm{C}$ in DMEM-10\% FBS until the staining in order to increase the survival of the cells.

Note: Alternatively, the cells can be stored at $4{ }^{\circ} \mathrm{C}$ in order to minimize the level of oxidative stress induced by cell homogenization. However, we noticed an increased level of apoptotic and dead cells with the incubation at $4^{\circ} \mathrm{C}$. 
15. Aliquots the cells in different tubes and stain the cells with the different staining solutions at $28^{\circ} \mathrm{C}$ in a thermoblock in the dark.

B. Oxidative stress (MitoSOX and CellROX Green), apoptosis and cell death staining

1. Centrifuge the cells for 2 min at $600 \times g$ at RT.

2. Remove supernatant and resuspend the cells in prewarmed $\mathrm{HBSS} / \mathrm{Ca} / \mathrm{Mg}$ at $28^{\circ} \mathrm{C}$.

3. Centrifuge the cells, remove the supernatant and stain the cells with $400 \mu \mathrm{l}$ of 2.5-5 $\mu \mathrm{M}$ solution of MitoSOX in HBSS/Ca/Mg for 15 min or a 1-2.5 $\mu \mathrm{M}$ solution of CellROX Green in DMEM-10\% FBS for 30 min at $28{ }^{\circ} \mathrm{C}$, in the dark. Wash the cells three times with $1 \mathrm{ml}$ of prewarmed $\mathrm{HBSS} / \mathrm{Ca} / \mathrm{Mg}$ to remove the excess of the probes.

4. To test the viability of the cells after homogenization, an Annexin V-APC and 7-AAD double staining can be performed in parallel for the oxidative stress staining. For Annexin V-APC and 7-AAD double staining cells were centrifuged, washed with $500 \mu \mathrm{l}$ of prewarmed $1 \mathrm{x}$ binding buffer at $28^{\circ} \mathrm{C}$ (from Annexin V-APC kit) and then incubated in $600 \mu \mathrm{l}$ of $1 \mathrm{x}$ binding buffer with $3 \mu \mathrm{l}$ of the probes for $15 \mathrm{~min}$ at $28^{\circ} \mathrm{C}$ in the dark. After the staining, wash the cells three times with $500 \mu \mathrm{l}$ of prewarmed $1 \mathrm{x}$ binding buffer.

5. Filter the cell solution through a sterile $40 \mu \mathrm{m}$ nylon mesh before to FACS analysis.

6. Transfer the cells to a $5 \mathrm{ml}$ tube and then sort the cells with a FACS.

7. Flow cytometry data were collected on a FACSAria II equipped with FACSDiva software using the blue 488-nm laser for 7-AAD (550/647 nm, Excitation/Emission peaks), CellROX green (CellROX green flow cytometry assay kit: 508/525 nm; CellROX green reagent: 485/520 nm) and MitoSOX (510/580 $\mathrm{nm}$ ) reagents and the red 640-nm laser (BD) for APC signal (650/660 $\mathrm{nm}$ ) (see Figure 2 for representative analysis).

C. Whole mount staining of ROS (MitoSOX and CellROX Green)

1. Grow zebrafish embryos at $28{ }^{\circ} \mathrm{C}$ in $\sim 25 \mathrm{ml}$ of fish water or $1 x \mathrm{E} 3$ embryo medium until the desired stage.

2. Collect the embryos and manually dechorionate them with forceps, if necessary.

3. Transfer the embryos to a $1.5 \mathrm{ml}$ tube (10-15 embryos/tube).

4. Prepare low-melting agarose solution using fish water and a specific amount of low-melting agarose powder according to the desired percentage of the gel using a microwave. Let it cool down for $5 \mathrm{~min}$ at RT then prepare some $1 \mathrm{ml}$ aliquots in $1.5 \mathrm{ml}$ tubes and keep them at $\sim 37^{\circ} \mathrm{C}$ in the thermoblock ready to be used (the percentage of agarose depends on the stage of the embryos: usually $0.5-0.8 \%$ until 1-2 dpf, $\sim 1 \%$ after $2 \mathrm{dpf}$ ).

5. Remove fish water or 1X E3 embryo medium and wash the embryos once in $1 \mathrm{ml}$ of $\mathrm{HBSS} / \mathrm{Ca} / \mathrm{Mg}$.

6. Remove the $\mathrm{HBSS} / \mathrm{Ca} / \mathrm{Mg}$ without damaging or drying the embryos and add the specific staining solution as previously indicated (see step B3). 
7. Incubate at $28^{\circ} \mathrm{C}$ in the dark. Usually $10-30$ min represents a good starting point, but the length of the incubation should be optimized based on the developmental stage, the staining solution and the oxidative status of the sample.

8. After the incubation time wash three times with $1 \mathrm{ml}$ of prewarmed $\mathrm{HBSS} / \mathrm{Ca} / \mathrm{Mg}$ at $28^{\circ} \mathrm{C}$, then anesthetize the embryos in $1 \mathrm{ml}$ of $1 \mathrm{x}$ tricaine in $1 \mathrm{x}$ E3 embryo medium for $\sim 5 \mathrm{~min}$.

9. Transfer the embryos individually in a $35 \mathrm{~mm}$ glass bottom dish, remove the excess of liquid (without damaging or drying the embryos) and mount them in $\sim 150 \mu$ l/embryo of low melting agarose gel. Orientate each embryo as desired using a small paintbrush under a dissecting microscope. After gel solidification, gently cover the embryos with enough volume of $1 x$ tricaine in 1x E3 embryo medium in order to anesthetize them (see Figure 1).

10. Analyze the embryos using a confocal microscope and a $488 \mathrm{~nm}$ laser. The MitoSOX and CellROX Green wavelengths of excitation and emission are: Mitosox 510/580 nm, CellROX green flow cytometry assay kit: 508/525 nm and CellROX green reagent: 485/520 nm (see Figure 2D).

11. After the imaging, the embryos can be recovered from the agarose using a pair of forceps in order to be genotyped, if necessary. 


\section{bĭ̈-protocol

A

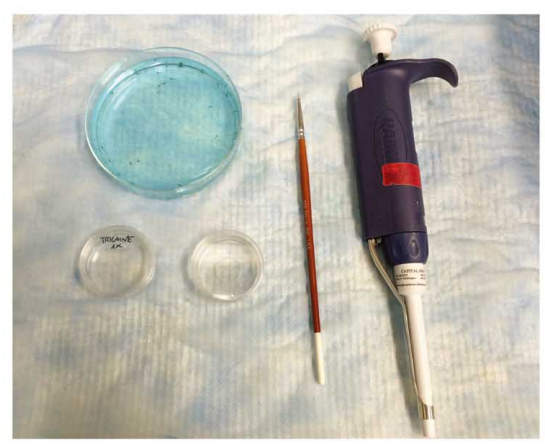

C

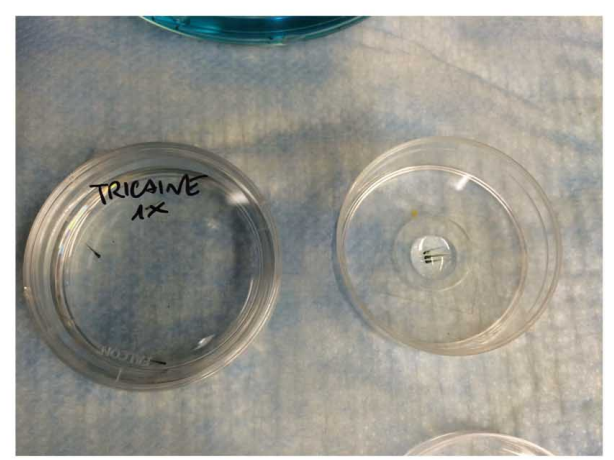

$\mathbf{E}$

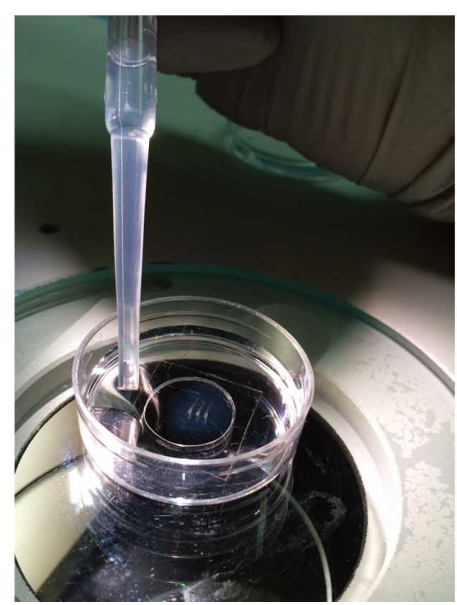

B
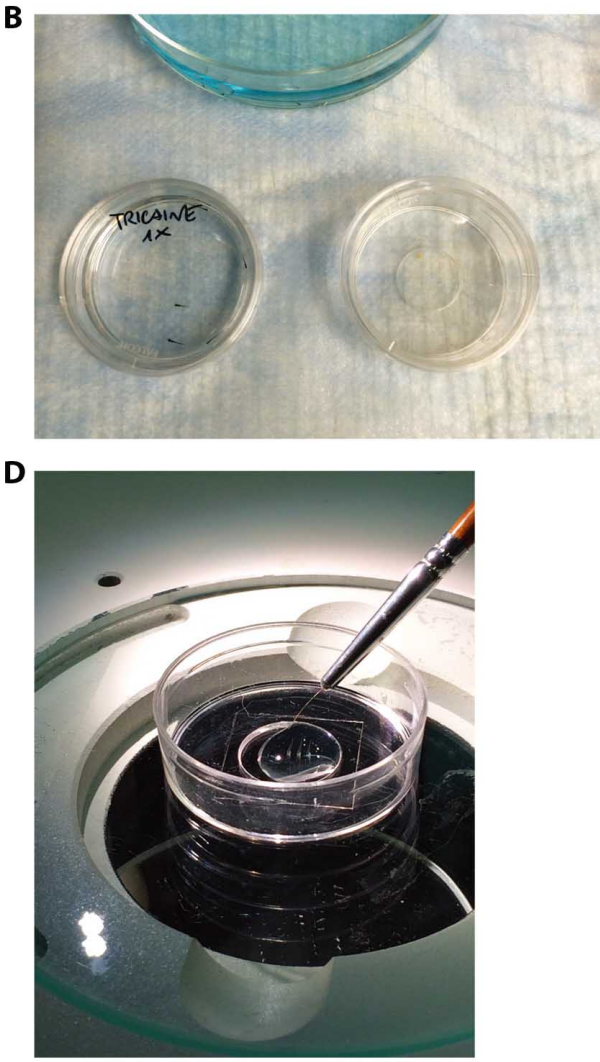

Figure 1. Mounting of live embryos in low melting agarose for confocal analysis. A. Tools required for zebrafish embryos inclusions in agarose gel. B. Prepare a small Petri dish with $1 \mathrm{x}$ tricaine in 1x E3 embryo medium in order to anesthetize the embryo (Petri dish on the left) and a $35 \mathrm{~mm}$ glass bottom dish (Petri dish on the right). Transfer 3 or more embryos in the anesthetic solution and wait until they are anesthetized. C. Transfer the anesthetized embryos to the 35 $\mathrm{mm}$ glass bottom dish and pour gently the agarose solution using a PR-200 pipette. D. Gently orientate the embryos using the small paintbrush without damaging them and until the agarose gel is solid enough to block them in the desired position. E. At the end of the solidification, the gel should appear slightly opaque and dense if touched using the paintbrush. In the specific case, $5 \mathrm{dpf}$ embryos have been orientated in a lateral position. Slowly fill the Petri dish with $1 \mathrm{x}$ tricaine in 1X E3 embryo medium in order to avoid the detachment of the gel from the glass. 


\section{Representative data}

A

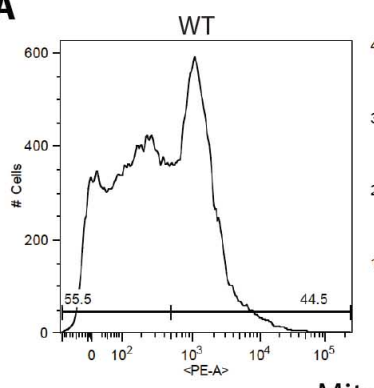

Mitosox

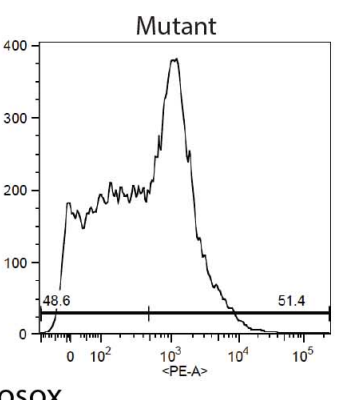

D
B

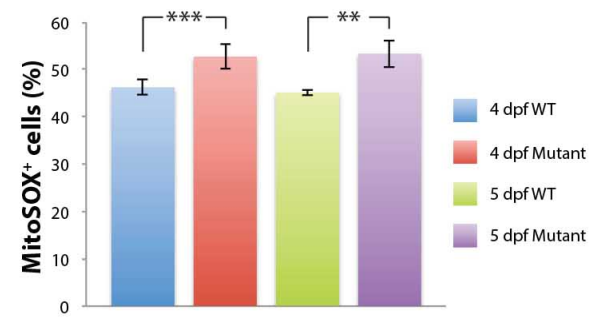

C

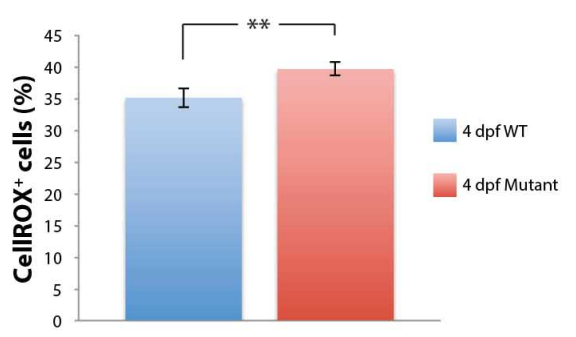

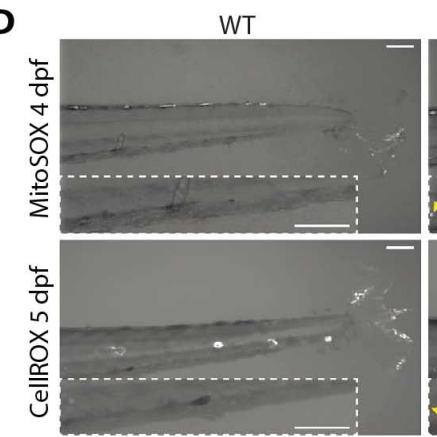

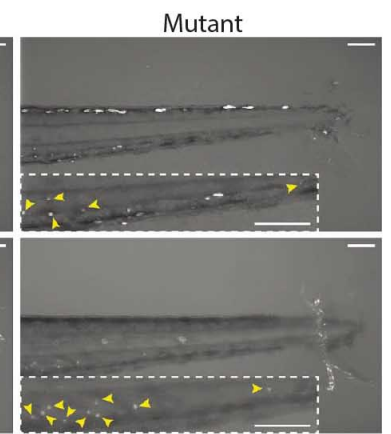

Figure 2. Representative analysis of oxidative stress in WT and mutant embryos using MitoSOX and CellROX staining. A-C. Representative quantitative analysis by flow cytometry of oxidative stress using MitoSOX Red (A and B) or CellROX Green (C) on 4 and 5 dpf mutant and control embryos. Error bars indicate standard deviation; ${ }^{\star \star} P<0.01 ;{ }^{\star \star \star} P<0.001$ using an unpaired Student's $t$-test. D. Whole mount staining with the MitoSOX or CellROX probes followed by confocal microscopy in the tail region of WT and mutant embryos at the indicated stages. As internal positive control, embryos at the stages of 4 or $5 \mathrm{dpf}$ were subjected to tail resection 30 min before being stained with MitoSOX or CellROX solutions. If tail resection cannot be performed, because of the stage analyzed or because it could interfere with the specific conditions of the experiment or the fish line used, an alternative positive control could be represented by the treatment of the embryos as indicated in Mugoni et al. (2014). Yellow arrowheads in the insets indicate MitoSOX- or CellROX-positive cells in CHT regions. CHT: Caudal Hematopoietic Tissue (from Rissone et al., 2015).

\section{$\underline{\text { Recipes }}$}

1. $24 x$ tricaine stock solution

$400 \mathrm{mg}$ tricaine powder in $97.9 \mathrm{ml} \mathrm{ddH_{2 } \mathrm { O }}$

Use $2.1 \mathrm{ml} 1 \mathrm{M}$ Tris (pH 9) to adjust $\mathrm{pH}$ to 7.

Store the solution in refrigerator for short period (1-2 weeks) or in the freezer in aliquots (1 month). 
Dilute to 1x with 1x E3 embryo medium (or fish water) to anesthetize the embryos.

2. DMEM/F-12 medium

Supplemented with $10 \%$ fetal bovine serum (FBS)

3. $60 x$ E3 embryo medium stock solution

$17.2 \mathrm{~g} \mathrm{NaCl}$

$0.76 \mathrm{~g} \mathrm{KCl}$

$2.9 \mathrm{~g} \mathrm{CaCl} 2 \cdot 2 \mathrm{H}_{2} \mathrm{O}$

$4.9 \mathrm{~g} \mathrm{MgSO}_{4} \cdot 7 \mathrm{H}_{2} \mathrm{O}$

Dissolve in $1 \mathrm{LddH} \mathrm{H}_{2} \mathrm{O}$

4. Homogenization solution

$10 \mathrm{mg} / \mathrm{ml}$ collagenase $\mathrm{P}$

$0.05 \%$ trypsin-EDTA

$1 \times$ PBS

5. $5 \mathrm{mM}$ MitoSOX stock solution

Dissolve the contents $(50 \mu \mathrm{g})$ of one vial of MitoSOX mitochondrial superoxide indicator in 13 $\mu \mathrm{l}$ of DMSO.

\section{Acknowledgments}

This protocol was modified from Behra et al. (2012) and Mugoni et al. (2014), and developed in the Dr. Fabio Candotti Lab. Disorders of Immunity Section, Genetics and Molecular Biology Branch, $\mathrm{NHGRI}, \mathrm{NIH}$. This protocol was supported by funding from the Intramural Research Program of the National Human Genome Research Institute (to F. Candotti, R. Sood, and S. Chandrasekharappa).

\section{References}

1. Behra, M., Gallardo, V. E., Bradsher, J., Torrado, A., Elkahloun, A., Idol, J., Sheehy, J., Zonies, S., Xu, L., Shaw, K. M., Satou, C., Higashijima, S., Weinstein, B. M. and Burgess, S. M. (2012). Transcriptional signature of accessory cells in the lateral line, using the Tnk1bp1:EGFP transgenic zebrafish line. BMC Dev Biol 12: 6.

2. Mugoni, V., Camporeale, A. and Santoro, M. M. (2014). Analysis of oxidative stress in zebrafish embryos. J Vis Exp(89).

3. Rissone, A., Weinacht, K. G., la Marca, G., Bishop, K., Giocaliere, E., Jagadeesh, J., Felgentreff, K., Dobbs, K., Al-Herz, W., Jones, M., Chandrasekharappa, S., Kirby, M., Wincovitch, S., Simon, K. L., Itan, Y., DeVine, A., Schlaeger, T., Schambach, A., Sood, R., Notarangelo, L. D. and Candotti, F. (2015). Reticular dysgenesis-associated AK2 protects hematopoietic stem and progenitor cell development from oxidative stress. J Exp Med 212(8): 1185-1202. 